\title{
Ratio of Serum Aspartate to Alanine Aminotransferase as a Marker of Isolated Coronary Artery Ectasia and its Severity
}

Danesh Soltani, ${ }^{1}$ Samira Jafari, ${ }^{1}$ Haleh Ashraf, ${ }^{2,3}$ Amir Sobh-Rakhshankhah, ${ }^{2}$ Zahra Kolahchi, ${ }^{1}$ Farzad Masoudkabir, ${ }^{2,4}$ Mohammad Ali Boroumand ${ }^{5}$ and Ali Vasheghani-Farahani ${ }^{2}$

1. Students' Scientific Research Center (SSRC), Tehran University of Medical Sciences, Tehran, Iran; 2. Cardiac Primary Prevention Research Center (CPPRC), Tehran Heart Center, Tehran University of Medical Sciences, Tehran, Iran; 3. Department of Cardiology, Sina Hospital, Tehran University of Medical Sciences, Tehran, Iran; 4. Department of Cardiology, Tehran Heart Center, Tehran University of Medical Sciences, Tehran, Iran; 5. Department of Pathology and Laboratory Medicine, Tehran Heart Center, Tehran University of Medical Sciences, Tehran, Iran

DOI: https://doi.org/10.17925/HI.2020.14.1.43

$\mathrm{B}$ ackground: Several studies have reported an association between elevated liver enzymes and increased risks for developing inflammatory diseases. The aim of our study was to examine how serum liver transaminases, as inexpensive and routinely measured markers, and the De Ritis ratio are associated with the presence of coronary artery ectasia (CAE) and its severity. Methods: Participants were recruited from patients admitted to Tehran Heart Center for diagnostic coronary angiography due to suspected myocardial ischaemia. These participants also underwent concurrent laboratory routine biochemical and liver enzyme tests. Results: A total of 104 participants were included; 59 had CAE and 45 were controls without coronary artery disease (CAD). The CAE group was split into a further two subgroups: those with isolated CAE $(n=27)$ and those with CAD and coexisting CAE $(n=32)$. In the adjusted multivariate analysis, a lower ratio of aspartate aminotransferase to ALT (AST/ALT) was, uniquely among the variables, a statistically significant marker for isolated CAE. In the CAD + CAE group, the AST/ALT ratio was not significant after adjustments for the confounding factors. The multivariate linear regression for the Markis score showed that the AST/ALT ratio was inversely associated with the severity of CAE. Conclusions: We conclude that the AST/ALT ratio and, to some extent, ALT independently of other inflammatory factors, can be associated with the presence and severity of isolated CAE.

\section{Keywords}

Transaminases, coronary artery ectasia,

coronary aneurysm, coronary artery disease, biomarkers

Disclosures: Danesh Soltani, Samira Jafari, Haleh Ashraf, Amir Sobh-Rakhshankhah, Zahra Kolahchi, Farzad Masoudkabir, Mohammad Ali Boroumand and

Ali Vasheghani-Farahani have no financial or non-financial

relationships or activities to declare in relation to this article.

Acknowledegments: The authors would like to thank all the participants and the staff of Tehran Heart Center. This study was a part of an MD thesis by Dr Samira Jafari and was supported by the Tehran University of Medical Sciences.

Review Process: Double-blind peer review.

Compliance with Ethics: All procedures were followed in accordance with the responsible committee on human experimentation and with the Declaration of Helsinki of 1975 and subsequent revisions, and informed consent was received from all patients involved in this study.

Data Sharing Statement: The data that support the fingings of this study are available from the Tehran Heart Center, but restrictions apply to the availability of

these data, which were used under license for the current study and so are not publicly available. Data are, however, available from the corresponding author on reasonable request, with the permission of the Tehran Heart Center.

Authorship: The named authors meet the International Committee of Medical Journal Editors (ICMJE) criteria for authorship of this

manuscript, take responsibility for the integrity of the work as a whole,

and have given final approval for the version to be published.

Access: This article is freely accessible at touchCARDIO.com

(C) Touch Medical Media 2020.

Received: 24 March 2020

Accepted: 27 April 2020

Published Online: 31 July 2020

Citation: Heart International. 2020;14(1):43-8

Corresponding Author: Ali Vasheghani-Farahani,

Tehran Heart Center, Karegar Shomali St, Jalal al-Ahmad

Intersection, Tehran, Iran. E: avasheghani@tums.ac.ir

Support: No funding was received for the publication of this article.
The exact aetiology of coronary artery ectasia (CAE) has yet to be fully elucidated; however, some underlying aetiologies such as atherosclerosis and microvascular abnormalities in coronary arteries have been proposed. ${ }^{1,2}$ Over the past few years, several inflammatory markers have been introduced as indicators of CAE and its severity. There is an association between the development of CAE and inflammatory markers such as high-sensitivity C-reactive protein (hs-CRP), uric acid, endocan, visfatin, interleukin-6 (IL-6) and white blood cells. ${ }^{3-6}$ Nonetheless, further research aimed at introducing novel predictable markers has been of great interest with a view to improving the early diagnosis of CAE. Liver transaminases are inexpensive inflammatory markers measured in a clinical setting. There has been a growing interest in studies on changes in liver enzymes in cardiovascular conditions due to the mutual interaction between the functions of the heart and the liver. Recent studies have demonstrated an association between elevated serum levels of liver enzymes - including alanine aminotransferase (ALT), aspartate aminotransferase (AST), the AST/ALT ratio (the De Ritis ratio), and gamma-glutamyl transferase (GGT) - and increased risks of inflammatory conditions (e.g., atherosclerosis and the metabolic syndrome $)^{7-9}$ and cardiovascular mortality. ${ }^{10}$ ALT has been known as a marker of non-alcoholic fatty liver disease, which is independently associated with cardiovascular events. ${ }^{11}$ There are, however, only a few studies investigating the predictive role of higher serum levels of liver enzymes, including alkaline phosphatase (ALP) and AST, in isolated $C A E,{ }^{12}$ and there is no study regarding the association between serum AST/ALT ratio and the development of CAE. Given the predictive value of serum liver enzymes in atherosclerosis and inflammatory processes, as well as the role of inflammation in the occurrence of CAE, we designed the present study to assess how liver transaminases and their ratio - as inexpensive and routinely measured markers - are associated with CAE. 


\section{Materials and methods} Study population

Participants were recruited from patients admitted to Tehran Heart Center. The study participants underwent diagnostic coronary angiography due to suspected myocardial ischaemia. CAE was defined as a 1.5- to 2-fold dilation of the coronary artery lumen in proportion to that of a normal diameter without stenosis. ${ }^{13}$ Those patients with CAE and atherosclerotic plaque in the coronary arteries were categorised as the coronary artery disease $(C A D)+C A E$ group, according to the definition of the American Heart Association. Participants in the control group were defined as those with no CAD or CAE.

Exclusion criteria were comprised of a history of myocardial infarction in the preceding 3 months; the use of drugs including statins, gemfibrozil, metformin, thiazolidinedione, corticosteroids and other drugs with anti-inflammatory actions in the preceding 3 months; a chronic consumption of alcohol; and a history of cancer, connective tissue diseases, hepatitis B and C, inflammatory bowel disease, haemodialysis, percutaneous coronary intervention and coronary artery bypass grafting.

All study participants were informed about the method and purpose of study and written informed consent was signed by each of them.

\section{Assessment of the risk factors and demographic data Demographic data and information about CAD risk factors were obtained through interviews and a structured questionnaire. Anthropometric indices - including weight, height, and waist circumference - were measured, and the body mass index (BMI) was calculated as weight $(\mathrm{kg})$ divided by the height squared $\left(\mathrm{m}^{2}\right)$. Systolic and diastolic blood pressure (SBP and DBP) were measured by an educated nurse using a sphygmomanometer with a blood-pressure cuff.}

Diabetes mellitus was defined as a minimum fasting blood glucose (FBG) level of $126 \mathrm{mg} / \mathrm{dL}(7.0 \mathrm{mmol} / \mathrm{L})$ or receiving antidiabetic treatments. ${ }^{14}$ Hypertension was defined as a blood pressure of at least 140/90 mmHg'15 or taking antihypertensive agents. Hyperlipidaemia was defined as fasting triglyceride (TG) levels $>150 \mathrm{mg} / \mathrm{dL}(1.7 \mathrm{mmol} / \mathrm{L})$ or total cholesterol (TC) levels $>200 \mathrm{mg} / \mathrm{dL}$ (5.2 mmol/L), or receiving lipid-lowering treatments. ${ }^{16}$

\section{Laboratory measurements}

The patients' blood samples were collected from the antecubital vein in Ethylenediaminetetraacetic acid (EDTA)-treated plain tubes for the baseline measurement of ALT, AST, GGT, FBG, high-density lipoprotein cholesterol (HDL-C), low-density lipoprotein cholesterol (LDL-C), total glycerides (TG) and total cholesterol (TC) via enzymatic methods. The AST/ALT ratio was calculated.

Glycated haemoglobin (HbA1C) was assessed using turbidimetric inhibition immunoassay with inter- and intra-assay coefficients of variation of $1.3 \%$ and $0.8 \%$ at a mean level of $5.3 \%(34 \mathrm{mmol} / \mathrm{mol})$ and $1.0 \%$ and $0.9 \%$ at a mean level of $10.2 \%$ ( $88 \mathrm{mmol} / \mathrm{mol})$, respectively, and a lower detection limit of $2.9 \%$. The serum hs-CRP concentration was measured via immunoturbidimetry using latex particles sensitized with specific antibodies with inter- and intra-assay coefficients of variation of $2.9 \%$ and $1.8 \%$ at a mean level of $0.62 \mathrm{mg} / \mathrm{dL}$ and $2.7 \%$ and $1.5 \%$ at a mean level of $14.2 \mathrm{mg} / \mathrm{dL}$, respectively, and a lower detection limit of $0.1 \mathrm{mg} / \mathrm{dL}$.

\section{Coronary angiography assessment}

Coronary angiography was carried out and analysed by two experienced cardiologists. Thereafter, based on the Markis categorisation, the patients were classified into four types of ectasia based on severity. Type I was defined as a diffuse ectasia of two or three vessels, type ॥l was diffuse disease in one vessel and a localised disease in another vessel, type III was a diffuse ectasia of one vessel only and type IV was a localised or segmental ectasia. ${ }^{17}$

\section{Statistical analysis}

Data analyses were performed using the IBM SPSS Statistics (version 16.0) and STATA (version 13). Normality of distribution was assessed using the Kolmogorov-Simonov test. Continuous (scale) variables with and without normal distributions were expressed as mean \pm standard deviations or median and interquartile ranges, correspondingly. Data with normal distribution were compared using the ANOVA test and data without normal distribution were compared using the Mann-Whitney $U$ and Kruskal-Wallis tests. Categorical variables were shown as percentages and compared using a $\chi^{2}$ test. If any of these tests showed significant difference between all three groups, post hoc tests were performed, using adjusted residual method for categorical variables and Scheffe test for continuous variables.

Values with non-normality were transformed. The correlation between liver transaminases (their total value and also their value in different tertile categories) and CAE was analysed in different models. Model I showed a univariate correlation with no adjustments, and in model II, the significant variables were adjusted in the univariate analysis. Multivariate logistic regression and linear regression analyses of the Ln-transformed values were utilised to evaluate the predictive value of liver transaminases and their ratio for the presence and severity of CAE. In addition, we checked the multicollinearity of all independent variables by examining the variance inflation factor (VIF) and tolerance factor. Although there is no agreement as to what VIF cut-off based value should be used to identify multicollinearity, the maximum acceptable level of VIF is 5 (or 10) in most studies. ${ }^{18,19}$ In our study, a tolerance of $<0.4$ or VIF of $>5$ was considered as multicollinearity. In our analysis, GGT, HDL and HDA1c showed considerable collinearity with the other independent variables, thus were removed from multiple regression. The other independent variables had acceptable values. Receiver operating characteristic (ROC) curves were determined and compared in order to identify the best predictor of CAE. A p value of $<0.05$ was considered statistically significant.

\section{Results}

Demographic, clinical and laboratory characteristics of study participants

The demographic, clinical and laboratory characteristics of the subjects are summarised in Tables 1 and 2. In total, 104 patients were enrolled and categorised into three groups: isolated CAE group ( $n=27), C A D+C A E$ group ( $n=32)$, and control group $(n=45)$. There was a significant difference among the three groups investigated regarding the sex distribution $(p<0.001)$ and hyperlipidaemia $(p<0.001)$; however, on post hoc analysis using adjusted residual test, only the CAD + CAE and control groups exhibited a significant difference between observed and expected counts, which is expected to be the main contributor to the overall chi-square value. The other demographic and baseline characteristics including age, height, weight, BMI, waist circumference, SBP, DBP and the presence of hypertension and diabetes mellitus - showed no significant differences as regards their prevalence rates. Among the laboratory findings, the serum levels of hs-CRP, LDL-C, and TC were significantly different among the three study groups $(p=0.006,0.022$ and 0.009 , respectively); however, in post hoc analysis, only the serum level of hs-CRP was significantly higher in the subjects with isolated CAE than in the control group $(p=0.008)$. Also, there was a significant difference in serum levels of LDL-C and TC between the isolated CAE and CAE + 
Table 1: Demographic and baseline characteristics of all study groups

\begin{tabular}{|c|c|c|c|c|}
\hline & $\begin{array}{l}\text { Isolated CAE } \\
(\mathrm{n}=27)\end{array}$ & $\begin{array}{l}\text { CAD + CAE } \\
(n=32)\end{array}$ & $\begin{array}{l}\text { Control } \\
(n=45)\end{array}$ & $P^{*}$ \\
\hline Age, years & $59.1 \pm 10.4$ & $58.2 \pm 10.5$ & $59.0 \pm 11.3$ & 0.939 \\
\hline $\begin{array}{l}\text { Sex } \\
\text { Male, n (\%) } \\
\text { Female, n (\%) }\end{array}$ & $\begin{array}{l}21(78) \\
6(22)\end{array}$ & $\begin{array}{l}29(91) \\
3(9)^{\dagger}\end{array}$ & $\begin{array}{l}21(47)^{\dagger} \\
24(53)^{\dagger}\end{array}$ & 0.0001 \\
\hline Height, cm & $167.04 \pm 9.90$ & $170.12 \pm 8.59$ & $166.50 \pm 14.85$ & 0.429 \\
\hline Weight, kg & $85.30 \pm 13.00$ & $83.31 \pm 9.68$ & $89.00 \pm 31.11$ & 0.701 \\
\hline $\mathrm{BMI}, \mathrm{kg} / \mathrm{m}^{2}$ & $30.70 \pm 5.27$ & $29.00 \pm 3.40$ & $31.50 \pm 5.60$ & 0.269 \\
\hline Waist, cm & $102.90 \pm 15.88$ & $104.19 \pm 13.30$ & $98.00 \pm 9.90$ & 0.817 \\
\hline $\mathrm{SBP}, \mathrm{mmHg}$ & $148.70 \pm 23.70$ & $154.80 \pm 27.70$ & $135.00 \pm 7.07$ & 0.440 \\
\hline $\mathrm{DBP}, \mathrm{mmHg}$ & $79.44 \pm 14.10$ & $81.45 \pm 12.40$ & $72.5 \pm 10.60$ & 0.594 \\
\hline HTN, n (\%) & $22(81)$ & $27(84)$ & $25(56)$ & 0.054 \\
\hline HLP, n (\%) & $19(70)$ & $12(38)^{\dagger}$ & $38(84)^{\dagger}$ & $<0.0001$ \\
\hline
\end{tabular}

*P value refers to the state of significance between all three groups.

tThese values (observed counts) are significantly higher/lower than expected counts, on post hoc test using standard residual method.

Data are presented as means \pm standard deviation unless stated otherwise.

$B M I=$ body mass index; $C A D=$ coronary artery disease; $C A E=$ coronary artery ectasia; $D B P=$ diastolic blood pressure; $D M=$ diabetes mellitus; $H L P=$ hyperlipidaemia; $H T N=$ hypertension; $S B P=$ systolic blood pressure.

\section{Table 2: Laboratory characteristics of all study groups}

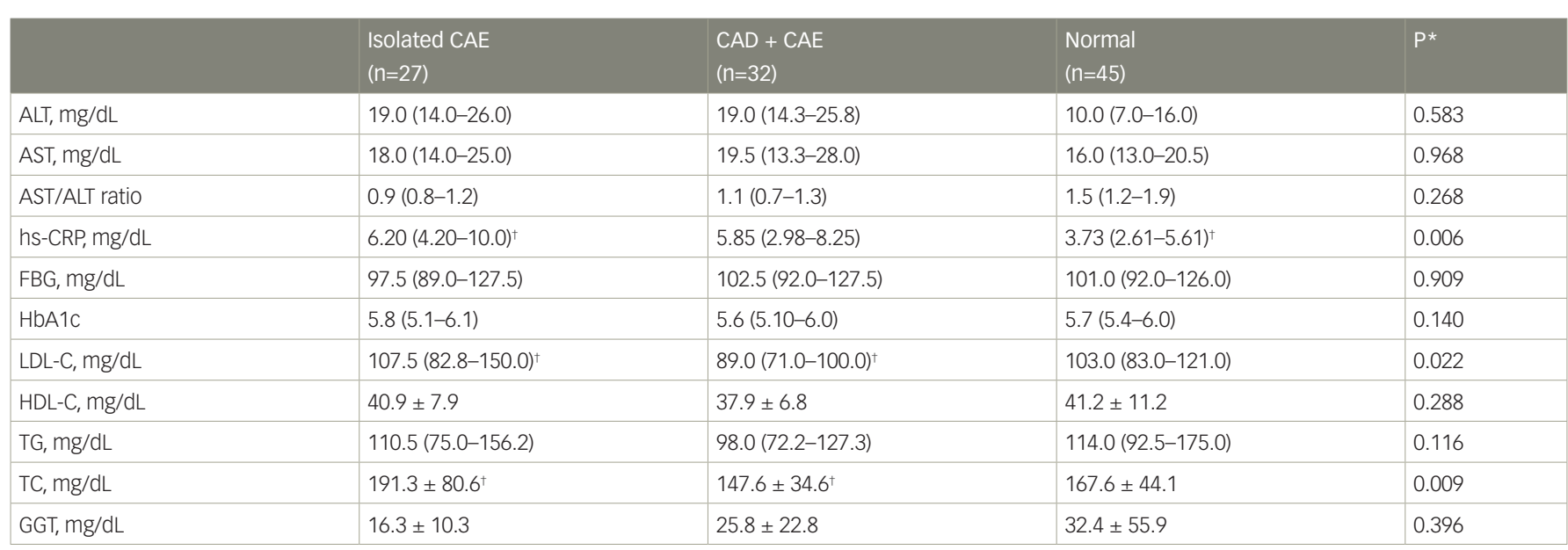

* $P$ value refers to the state of significance between all three groups.

tThis refers to study groups with significant difference from each other, on post hoc Scheffe test.

Data are presented as median (interquartile ranges) or mean \pm standard deviation.

ALT = alanine transaminase; $A S T=$ aspartate transaminase; $C A D=$ coronary artery disease; $C A E$ = coronary artery ectasia; FBG = fasting blood glucose; GGT = gamma-g/utamyl transferase; $H \mathrm{HDA} 1 \mathrm{C}=$ glycated haemoglobin; $H D L-C=$ high-density lipoprotein cholesterol; $h$ s-CRP = high-sensitive C-reactive protein; $L D L-C=$ low-density lipoprotein cholesterol; $T C=$ total cholesterol; $T G=$ triglyceride.

CAD group ( $p=0.023$ and 0.010 , respectively). There were no statistically significant differences between the groups apropos of serum levels of AST $(p=0.968)$ and ALT $(p=0.583)$.

\section{Angiographic characteristics in both CAE groups}

The most involved arteries were the left anterior descending artery $(92 \%)$ and the right coronary artery (78\%) in the group with isolated CAE, and the left anterior descending artery (84\%) and the left circumflex artery (71\%) in the CAD + CAE group (Figure 1). Based on the Markis classification of ectasia, the patients with ectasia type II formed the most common group in our study (67\% in the isolated CAE group and $43 \%$ in the CAD + CAE group; Figure 2).

\section{Multivariate analysis of the variables}

The multivariate correlation analysis of the variables is summarised in Table 3. The predictive value of the variables for the group with isolated
CAE and the CAD + CAE group was analysed in different models. In the group with isolated CAE, the serum level of AST showed no significant association with CAE in the crude model $(p=0.812)$ and remained non-significant after adjustments for the mentioned factors $(p=0.653)$. A significant positive association was observed between the serum level of ALT and CAE in the univariate analysis (odds ratio [OR] 2.21; $95 \%$ confidence interval [Cl] 1.08-4.52; $\mathrm{p}=0.034$ ); however, this association was not statistically significant after adjustments for the confounding factors (OR 3.20; 95\% Cl 0.89-11.56; $p=0.074$ ). The AST/ALT ratio was independently and inversely associated with isolated CAE (OR 0.22; 95\% $\mathrm{Cl}$ 0.53-0.89; $\mathrm{P}=0.033$ ). The levels of hs-CRP, LDL and TC were not statistically significant predictors of isolated CAE after adjustments for the confounding factors. All the other markers - including GGT, HDA1C, $\mathrm{FBG}, \mathrm{HDL}$, and $\mathrm{TG}$ - were not statistically significant in either model. In the CAD + CAE group, no significant or independent association was found between the serum level of AST and CAE. In model I, 
Figure 1: Distribution of ectatic arteries in the group with isolated CAE and the CAD + CAE group

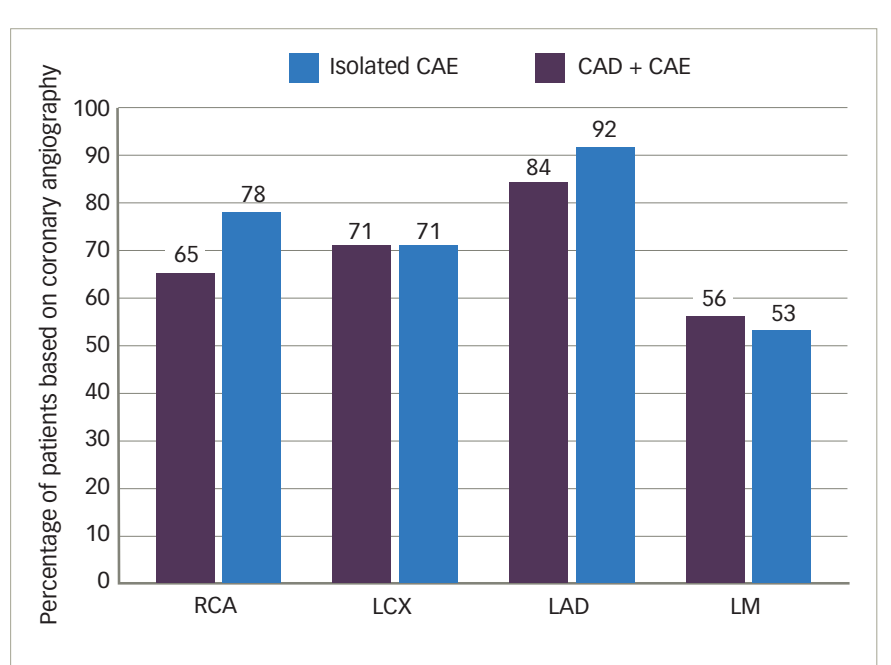

The LAD was the most involved artery in both the group with isolated CAE (92\%) and the CAD + CAE (84\%) group.

$C A D=$ coronary artery disease $: C A E=$ coronary artery ectasia; $L A D=$ left anterior descending artery; $L C X=$ left circumflex artery; $L M=$ left main artery; $R C A=$ right coronary artery.

Figure 2: Distribution of the different types of CAE according to the Markis categorisation in the group with isolated CAE and the CAD + CAE group

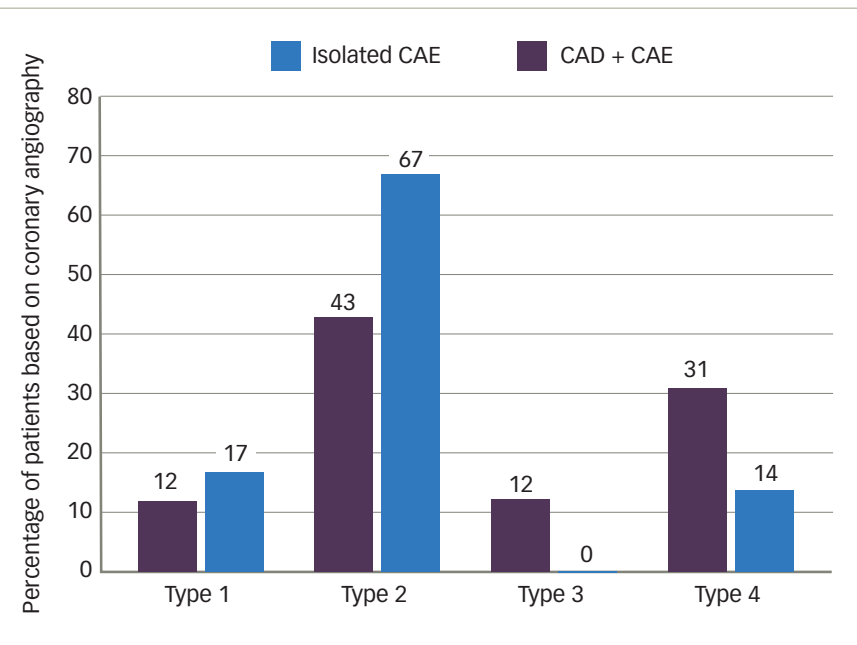

Patients with ectasia type II formed the most common group (67\% in the group with isolated CAE and $43 \%$ in the CAD + CAE group).

$C A D=$ coronary artery disease $; C A E=$ coronary artery ectasia

ALT level and AST/ALT ratio had a significant correlation with CAE (OR 2.07; 95\% Cl 1.04-4.11; $\mathrm{p}=0.047$ and OR 0.42; 95\% Cl 0.18-0.99; $\mathrm{p}=0.042$, respectively); however, this correlation was non-significant after adjustments for the confounding factors in model $\|$ ( $p=0.682$ and 0.783 , respectively). An analysis was also conducted by dividing study participants into three groups according to tertiles of AST and $A L T$, but the results remained non-significant. The levels of $L D L, T C$ and TG demonstrated no significant or independent correlations in the multivariate analysis. All the other variables had no significant associations in either model.

Multivariate linear regression for the assessment of ectasia severity

The multivariate linear regression for the Markis score according to the logarithm of ALT, AST and the AST/ALT ratio is summarised in Table 4.
Figure 3: ROC analysis to determine the best predictor value in the group with isolated $\mathrm{CAE}$

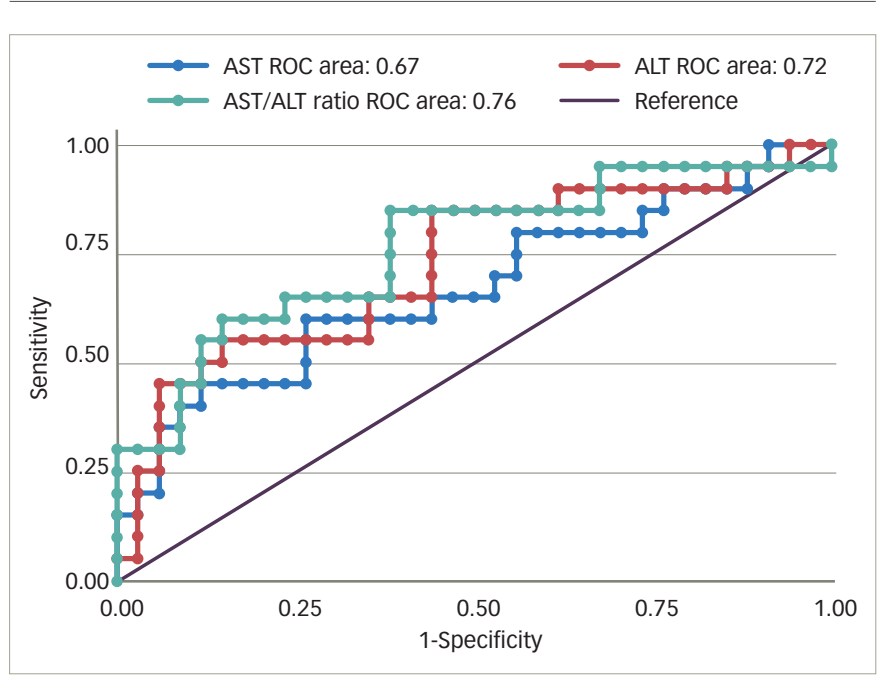

The AST/ALT ratio (AUC 0.76; 95\% Cl 0.62-0.90; $p=0.03$ ) was a significant predictor of isolated CAE. The predictive value of ALT and AST was not statistically significant. The statistical comparison between the ROC curves showed no significant difference $(p=0.40)$.

$A L T=$ alanine transaminase $; A S T=$ aspartate transaminase $; A U C=$ area under the curve; $C A E$ = coronary artery ectasia; $\mathrm{Cl}=$ confidence interval; $R O C=$ receiver operating characteristic.

Figure 4: ROC analysis to determine the best predictor value in the CAD + CAE group

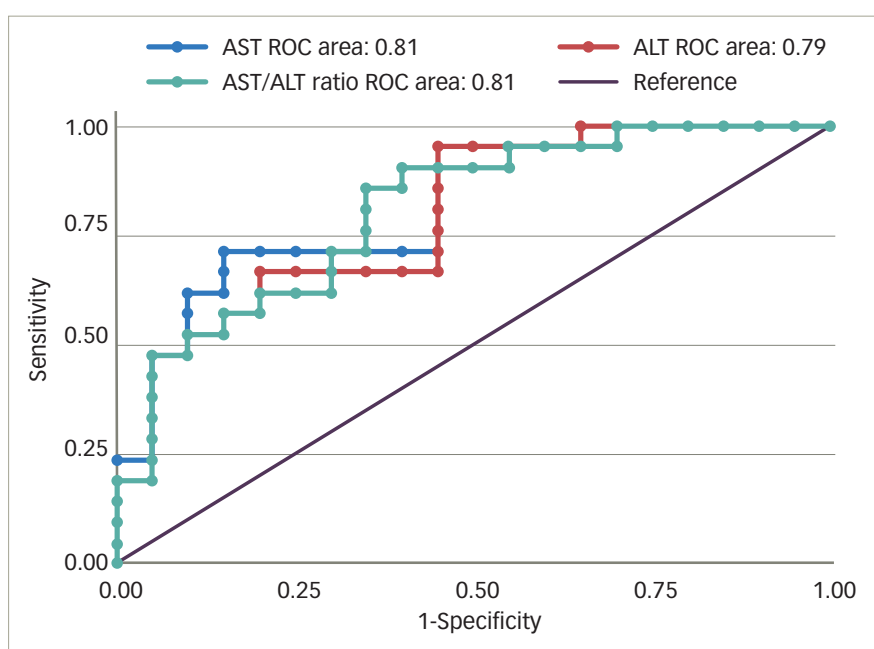

There was no significant association between liver transaminases and CAE. The statistical comparison between the ROC curves showed no significant difference $(p=0.71)$

$A L T=$ alanine transaminase $: A S T=$ aspartate transaminase: $A U C=$ area under the curve; $C A D=$ coronary artery disease; $C A E=$ coronary artery ectasia; $\mathrm{Cl}=$ confidence interval; $R O C=$ receiver operating characteristic.

The serum value of ALT was positively associated with the severity of CAE in the crude model (B 0.24; 95\% Cl 0.10-0.94; $\mathrm{P}=0.021)$ and close to being statistically significant after adjustments for the confounding variables (B $0.23 ; 95 \% \mathrm{Cl}-0.10$ to $0.94 ; \mathrm{p}=0.051$ ). On the other hand, the AST/ALT ratio showed an inverse and independent association with the severity of CAE (B -0.25; $95 \% \mathrm{Cl}-1.08$ to $0.06 ; \mathrm{p}=0.032$ ).

\section{ROC analysis to determine the predictive values of liver transaminases}

Figures 3 and 4 illustrate the ROC curves of liver transaminases for the group with isolated CAE and the CAD + CAE group, respectively. In patients with isolated CAE, only the AST/ALT ratio was a significant indicator of CAE (area under the curve [AUC] 0.76; 95\% Cl 0.62-0.90; $\mathrm{p}=0.033$ ). 
Table 3: Logistic regression analysis for development of CAE according to laboratory markers

\begin{tabular}{|c|c|c|c|c|c|c|c|c|}
\hline \multirow{3}{*}{ Variable } & \multicolumn{4}{|l|}{ Isolated CAE } & \multicolumn{4}{|l|}{$C A D+C A E$} \\
\hline & \multicolumn{2}{|l|}{ Model I } & \multicolumn{2}{|l|}{ Model II } & \multicolumn{2}{|l|}{ Model I } & \multicolumn{2}{|l|}{ Model II } \\
\hline & OR $(95 \% \mathrm{Cl})$ & $\mathrm{P}$ & OR $(95 \% \mathrm{Cl})$ & $P$ & OR $(95 \% \mathrm{Cl})$ & $P$ & OR $(95 \% \mathrm{Cl})$ & $\mathrm{P}$ \\
\hline $\mathrm{AST}^{*}$ & $1.12(0.45-2.81)$ & 0.812 & $\begin{array}{l}0.71(0.17-3.07) \\
\text { T1: Reference group } \\
\text { T2 :0.38 (0.08-1.92) } \\
\text { T3: } 0.61(0.14-2.71)\end{array}$ & $\begin{array}{l}0.653 \\
0.240 \\
0.512\end{array}$ & $1.40(0.59-3.35)$ & 0.442 & $\begin{array}{l}1.63(0.45-5.88) \\
\text { T1: Reference group } \\
\text { T2: } 1.50(0.33-6.72) \\
\text { T3: } 1.23(0.30-5.01)\end{array}$ & $\begin{array}{l}0.450 \\
0.601 \\
0.776\end{array}$ \\
\hline $\mathrm{ALT}^{*}$ & $2.21(1.08-4.52)$ & 0.034 & $\begin{array}{l}3.20 \text { (0.89-11.56) } \\
\text { T1: Reference group } \\
\text { T2: } 1.85(0.28-12.20) \\
\text { T3: } 4.12(0.61-27.94)\end{array}$ & $\begin{array}{l}0.074 \\
0.520 \\
0.153\end{array}$ & $2.07(1.04-4.11)$ & 0.047 & $\begin{array}{l}\text { 1.24 (0.44-3.53) } \\
\text { T1: Reference group } \\
\text { T2: } 0.58(0.12-2.76) \\
\text { T3: } 1.15(0.27-4.98)\end{array}$ & $\begin{array}{l}0.682 \\
0.501 \\
0.856\end{array}$ \\
\hline AST/ALT* & $0.21(0.08-0.61)$ & 0.004 & $0.22(0.53-0.89)$ & 0.033 & $0.42(0.18-0.99)$ & 0.042 & $1.16(0.40-3.31)$ & 0.783 \\
\hline GGT & $0.95(0.91-1.01)$ & 0.085 & - & - & $1.01(0.99-1.04)$ & 0.412 & - & - \\
\hline hs-CRP* & $3.04(1.24-7.41)$ & 0.010 & 2.85 (0.84-9.65) & $0.095^{\dagger}$ & $1.38(0.63-3.01)$ & 0.420 & - & - \\
\hline $\mathrm{HbA} 1 \mathrm{C}^{*}$ & $0.24(0.01-4.02)$ & 0.324 & - & - & $0.20(0.01-2.98)$ & 0.246 & - & - \\
\hline $\mathrm{FBG}^{*}$ & $0.95(0.21-4.37)$ & 0.950 & - & - & $0.93(0.22-3.96)$ & 0.927 & - & - \\
\hline $\mathrm{LDL}^{*}$ & $3.82(1.10-13.22)$ & 0.035 & $0.500 .01-16.90)$ & $0.701^{\dagger}$ & $0.29(0.09-0.96)$ & 0.040 & $0.11(0.00-5.73)$ & 0.285 \\
\hline $\mathrm{HDL}$ & $1.01(0.96-1.06)$ & 0.714 & - & - & $0.96(0.92-1.01)$ & 0.135 & - & - \\
\hline TC & $1.01(1.00-1.02)$ & 0.032 & $1.01(0.99-1.03)$ & $0.520^{\dagger}$ & $0.98(0.97-0.99)$ & 0.010 & $1.00(0.97-1.03)$ & 0.990 \\
\hline $\mathrm{TG}^{*}$ & $1.00(0.40-2.54)$ & 0.991 & - & - & $0.33(0.12-0.91)$ & 0.034 & $0.65(0.18-2.26)$ & 0.491 \\
\hline
\end{tabular}

* Ln-transformed values.

${ }^{+}$ALT and the AST/ALT ratio have been added to Model II.

Data are presented as OR (95\% CI). Model I: crude (univariate). Model II: adjusted for sex, HLP, TC, LDL, hs-CRP (only in the patients with isolated CAE) and TG (only in the patients with $C A D)$.

$A L T=$ alanine transaminase; $A S T=$ aspartate transaminase $; C A D=$ coronary artery disease; $C A E=$ coronary artery ectasia; $C l=$ confidence interval; $F B G=$ fasting blood glucose GGT = gamma-g/utamyl transferase; HDA1C = glycated haemoglobin; HDL-C = high-density lipoprotein cholesterol; HLP = hyperlipidaemia; $h$ s-CRP = high-sensitive C-reactive protein; $L D L-C=$ low-density lipoprotein cholesterol; $O R=$ odds ratio; $T=$ tertile; $T C=$ total cholesterol; $T G=$ triglyceride.

Table 4: Multivariate linear regression for the Markis score according to the logarithm of ALT and AST

\begin{tabular}{|l|l|l|l|l|}
\hline Variable & Model I & \multicolumn{3}{l|}{ Model II } \\
\hline & Beta & $p$ & Beta & $p$ \\
\hline AST* & $0.04(-0.47$ to 0.71$)$ & 0.684 & $0.04(-0.60$ to 0.82$)$ & 0.756 \\
\hline ALT* & $0.24(0.10$ to 0.94$)$ & 0.021 & $0.23(-0.01$ to 1.09$)$ & 0.051 \\
\hline AST/ALT* & $-0.3(0.08$ to 0.61$)$ & 0.001 & $-0.25(-1.08$ to 0.06$)$ & 0.032 \\
\hline
\end{tabular}

\section{* Ln-transformed values.}

Data are presented as standardised beta coefficients (95\% Cl). Model I: crude

(univariate). Model II: adjusted for sex, HLP, TC, LDL, hS-CRP (only in the patients with isolated CAE).

$A L T=$ alanine transaminase; $A S T=$ aspartate transaminase; $H L P=$ hyperlipidaemia; hs-CRP = high-sensitive C-reactive protein; $L D L-C=$ low-density lipoprotein

cholesterol; $T C=$ total cholesterol.

The predictive values of ALT and AST were not statistically significant. In the CAD + CAE group, none of the variables showed a significant correlation for the development of CAE. The AUC for the AST/ALT ratio was greater than the AUC for AST and ALT. Nevertheless, after the ROC comparative analysis (Figures 3 and 4), the comparisons of the AUCs of the predictors were almost identical and showed no significant differences with each other in both groups.

\section{Discussion}

This study demonstrated that higher serum levels of the AST/ALT ratio were significantly and inversely correlated with the presence of ectasia in patients with isolated CAE independent of the other confounding factors. The correlation between ALT and AST with the presence of isolated CAE were not statistically significant. Furthermore, we concluded that ALT and the AST/ALT ratio were independent markers of the severity of CAE compared with the control group.
The exact pathogenesis of ectasia has not yet been identified, although its coexistence with atherosclerosis and inflammatory processes has been proposed. Indeed, several studies have suggested the role of inflammatory markers in the prediction of CAE. ${ }^{20}$ In addition, the high mortality rate in patients suffering from CAD with simultaneous CAE in comparison with patients without ectasia provides further stimulus for researchers to identify useful markers for the prediction of CAE. ${ }^{21}$ Recent investigations have suggested that CAE is a systemic condition of the vascular wall rather than a local abnormality. ${ }^{20}$ Despite the higher rate of the coexistence of CAE with CAD, several differences have been demonstrated between them regarding their inflammatory biomarkers. Thus, isolated CAE should be investigated independently of CAD to obtain further information about its underlying mechanism. ${ }^{20,22}$

Liver transaminases, including AST and ALT, are widely used and routinely measured markers in a clinical setting. Previous studies have suggested that increased serum levels of ALT and AST are independently associated with atherosclerosis and cardiovascular events., 8,1,23 There is also some evidence suggesting that the AST/ALT ratio is an independent predictor of coronary atherosclerosis. ${ }^{8}$ However, to the best of our knowledge, the association between liver transaminases and CAE has been scarcely investigated. Additionally, no study has examined the relationship between the AST/ALT ratio and CAE. Huang et al. reported results in conflict with those in our study, insofar as they demonstrated that whereas higher serum levels of ALP activity and AST (elevated at inflammatory status) were associated with the presence of isolated CAE, there was no such association concerning the level of ALT. ${ }^{22}$ We found no association between AST and ALT with CAE in our subjects with isolated CAE and CAD coexisting with CAE. Our findings demonstrated that a lower ratio of AST/ALT was significantly associated with a higher rate of isolated CAE. We detected no significant association between CAE and 
the level of ALT and the AST/ALT ratio in the CAD + CAE group. These discrepancies between the results of the two groups can be explained by several distinct differences in the pathophysiology of isolated CAE and CAD. As was explained previously, the inflammatory process in CAE is beyond a localised atherosclerotic process. Additionally, high serum levels of several inflammatory biomarkers - including interferon-gamma, tumour necrosis factor- $\alpha, \mathrm{IL}-1 \beta, \mathrm{IL}-6$, and IL-8 - in CAE compared with those in CAD may be another reason for these different results. ${ }^{22}$

There might be some other probable hypothetical mechanisms for our findings. For example, matrix metalloproteinases (MMPs) are a large group of endopeptidases that have various functions. Degradation of the extracellular matrix is their main function, but they can also affect cytokines and chemokines and have a regulatory role in immunity and inflammation. Serum levels of MMPs increase in acute and chronic liver injuries. Different subtypes of MMPs have specific roles in liver pathologies like liver fibrogenesis, hepatocellular carcinoma, cirrhosis and chronic hepatitis. ${ }^{24-26}$ On the other hand, studies show that some subtypes of MMPS, such as MMP-9 and MMP-3, are associated with CAE. ${ }^{27,28}$ Serum levels of these MMPs have higher ratios in patients with CAE and also correlate with the severity of CAE. Degradation of the extracellular matrix and thus loosening the connective tissue could be the underlying mechanism. ${ }^{27,28}$ Therefore, it can be hypothesised that liver injuries (increased rates of AST and ALT), and subsequent expression of MMPs, are associated with a higher risk of CAE in patients.

Also, several studies have shown that ALT and the AST/ALT ratio are strongly associated with the prevalence of metabolic syndrome. ${ }^{29,30}$ Based on cohort studies, elevated ALT and decreased AST/ALT ratio are significant predictors of incident metabolic syndrome and its components, including high levels of TG and low levels of HDL-C and TC. ${ }^{31,32}$ The certain mechanism of this association is still unclear; however, one possible biologic pathway is increasing hepatic fat in the liver, causing liver enzyme elevation and also systemic insulin resistance, which independently provokes all components of metabolic syndrome. ${ }^{30}$ On the other hand, Doğduş et al. showed that atherogenic indices, shown to be associated with liver transaminases, have some possible roles in the pathologic process of isolated CAE..$^{33}$ This evidence of common pathogenesis of metabolic syndrome and isolated CAE may indicate how liver enzyme levels are associated with isolated CAE. Accumulation of pericardial fat may be another a probable common pathogenesis of metabolic syndrome and isolated CAE. ${ }^{34}$

As mentioned above, atherosclerotic lesions and CEA have common pathophysiology. Studies have shown that one of the cytokines associated with these mechanisms is IL-17; patients with isolated CEA had higher serum levels of IL-17 compared with controls. ${ }^{35,36}$ Increased IL-17 serum level is also an indicator of liver injuries and induces inflammation processes in the liver which cause the release of AST and ALT into blood circulation. Inflammation and proinflammatory cytokines are also one of the underlying aetiologies of isolated CEA. It could be hypothesised that IL-17 is the biochemical link between isolated CEA and liver enzymes. ${ }^{36-38}$

\section{Limitations}

First, because the patients enrolled in our study were candidates for coronary angiography, our findings cannot be extrapolated to the general population. Second, given the cross-sectional design of our study, the causality of the association cannot be determined. Third, we used a single baseline measurement rather than several measurements at different time points. We, therefore, cannot evaluate the effects of changes in these parameters; however, these patients should be monitored in order to evaluate the pattern of changes in markers over time, and to declare a cause and effect relationship. Finally, the observational design of this study precludes us from making conclusions with regard to event outcomes.

\section{Conclusion}

Our results reinforce the evidence that serum transaminase, as an inexpensive and routinely measured marker, can be used as an indicator of inflammation status and specifically demonstrated that high ALT level and low AST/ALT ratio, independent of other inflammation factors, are associated with the presence and severity of isolated CAE. $\square$
1. Demir M, Demir $C$, Keçeoğlu S. The relationship between serum bilirubin concentration and coronary artery ectasia. Postepy Kardiol Interwencyinej. 2015;11:202-5

2. Demir S, Avsar MK, Karakaya Z, et al. Increased mean platelet volume is associated with coronary artery ectasia. Postepy Kardiol Interwencyjnej. 2013;9:241-5.

3. Li J-J, Nie S-P, Qian X-W, et al. Chronic inflammatory status in patients with coronary artery ectasia. Cytokine. 2009;46:61-4.

4. Turan T, Akyuz AR, Aykan AC, et al. Plasma endocan levels in patients with isolated coronary artery ectasia. Angiology. 2016;67:932-6.

5. Demir Ş, Karakoyun G, Kanadasi M. Elevated high sensitivity $\mathrm{C}$-reactive protein and uric acid levels in coronary artery ectasia. Acta Biochimica Polonica. 2014;61:687-91.

6. Ashraf H, Soltani D, Sobh-Rakhshankhah A, et al. Visfatin as marker of isolated coronary artery ectasia and its severity. marker of isolated coronary
Cytokine 2019:113:216-20.

7. Yamada J, Tomiyama $\mathrm{H}$, Yambe $\mathrm{M}$, et al. Elevated serum levels of alanine aminotransferase and gamma glutamyltransferase are markers of inflammation and oxidative stress independent of the metabolic syndrome. Atherosclerosis. 2006;189:198-205.

8. Adibi P, Sadeghi M, Mahsa M, et al. Prediction of coronary atherosclerotic disease with liver transaminase level. Liver Int. 2007;27:895-900.

9. Masoudkabir F, Karbalai S, Vasheghani-Farahani A, et al. The association of liver transaminase activity with presence and severity of premature coronary artery disease. Angiology. 2011;62:614-9.

10. Yokoyama M, Watanabe $\mathrm{T}$, Otaki $\mathrm{Y}$, et al. Association of the aspartate aminotransferase to alanine aminotransferase ratio aspartate aminotransferase to alanine aminotransferase ratio
with BNP level and cardiovascular mortality in the general population: the Yamagata Study 10-year follow-up. Dis Markers. population: the Yam

11. Schindhelm RK, Diamant M, Dekker JM, et al. Alanine aminotransferase as a marker of non-alcoholic fatty liver disease in relation to type 2 diabetes mellitus and cardiovascular disease Diabetes Metab Res Rev. 2006;22:437-43.

12. Huang QJ, Guo YL, Zhu CG, et al. Association of alkaline phosphatase with isolated coronary artery ectasia. Scand 1 Clin Lab Invest. 2014;74:228-34.
13. Swaye $P S$, Fisher $L D$, Litwin $P$, et al. Aneurysmal coronary artery disease. Circulation. 1983;67:134-8.

14. American Diabetes Association. Standards of medical care in diabetes-2014. Diabetes Care. 2014;37(Suppl. 1):S14-80.

15. Gabb GM, Mangoni AA, Anderson CS, et al. Guideline for the diagnosis and management of hypertension in adults-2016. Med J Aust. 2016;205:85-9.

16. Expert Panel on Detection, Evaluation, and Treatment of High Blood Cholesterol in Adults. Executive summary of the Third Report of the National Cholesterol Education Program (NCEP) expert panel on detection, evaluation, and treatment of high blood cholesterol in adults (Adult Treatment Panel III). JAMA. 2001;285:2486-97

17. Markis JE, Joffe $C D$, Cohn PF, et al. Clinical significance of coronary arterial ectasia. Am I Cardiol. 1976:37.217-22.

18. Hair Jr JF, Black WC, Babin BJ, Anderson RE. Multivariate Data Analysis Seventh Edition, Harlow: Pearson Education Limited 2014;197-201.

19. Kutner MH, Nachtsheim CJ, Neter J, Li W. Applied Linear Statistical Models, Fifth Edition, New York: McGraw-Hill Irwin, 2005;408-9.

20. Yetkin $\mathrm{E}$, Waltenberger J. Novel insights into an old controversy: is coronary artery ectasia a variant of coronary atherosclerosis? Clin Res Cardiol. 2007;96:331-9.

21. Shakerian F, Sanati H, Kiani R, et al. Comparison of outcomes of diseased coronary arteries ectasia, stenosis and combined. Res Cardiovasc Med. 2015;4:e25206.

22. Yetkin $\mathrm{E}, \mathrm{Ozturk} \mathrm{S}$, Yetkin $\mathrm{G}$. Inflammation in coronary artery ectasia compared to atherosclerosis. Int J Mol. 2018;19:2971.

23. Schindhelm RK, Dekker JM, Nijpels G, et al. Alanine aminotransferase predicts coronary heart disease events: a 10-year follow-up of the Hoorn Study. Atherosclerosis. a 10-year follow-up
2007:191:391-6.

24. Ando W, Yokomori $H$, Tsutsui $N$, et al. Serum matrix metalloproteinase-1 level represents disease activity as oppose to fibrosis in patients with histologically proven nonalcoholic steatohepatitis. Clin Mol Hepatol. 2018;24:61-76.

25. Duarte S, Baber J, Fujii T, Coito AJ. Matrix metalloproteinases in liver injury, repair and fibrosis. Matrix Biol. 2015;44-6:147-56.

26. Roeb E. Matrix metalloproteinases and liver fibrosis (translational aspects). Matrix Biol. 2018;68-9:463-73.
27. Ammar W, Kappary M, Baghdady Y Shehata M. Matrix metalloproteinase-9 (MMP9) and high sensitivity C-reactive protein (hs-CRP) in coronary artery ectasia. Egypt Heart J. 2014;66(Suppl. 1):15

28. Dogan A, Tuzun N, Turker Y, et al. Matrix metalloproteinases and inflammatory markers in coronary artery ectasia: their relationship to severity of coronary artery ectasia. Coron Artery Dis. 2008;19:559-63.

29. Kim HR, Han MA. Association between serum liver enzymes and metabolic syndrome in Korean adults. Int J Environ Res Public Health. 2018;15:1658.

30. Lee $\mathrm{K}$, Yang JH. Which liver enzymes are better indicators of metabolic syndrome in adolescents: the Fifth Korea National Health and Nutrition Examination Survey, 2010. Metab Syndr Relat Disord. 2013;11:229-35.

31. Hanley AJ, Williams K, Festa A, et al. Liver markers and development of the metabolic syndrome: the insulin resistance atherosclerosis study. Diabetes. 2005;54:3140-7.

32. Yadav D, Choi E. Incremental predictive value of serum AST-to-ALT ratio for incident metabolic syndrome: the ARIRANG study. PLOS One. 2016;11:e0161304.

33. Doğduş M, Güler T. Investigation of atherogenic dyslipidemia in patients with isolated coronary artery ectasia. Kocaeli Med J. 2019;8:173-81.

34. Yang JJ, Yang $X$, Chen ZY, et al. Prevalence of coronary artery ectasia in older adults and the relationship with epicardial fat volume by cardiac computed tomography angiography. J Geriatr Cardiol. 2013;10:10-5.

35. Erbel C, Chen L, Bea F, et al. Inhibition of IL-17A attenuates atherosclerotic lesion development in ApoE-deficient mice I Immunol. 2009:183:8167.

36. Uygun T, Demir B, Tosun V, et al. Relationship between interleukin-17A and isolated coronary ectasia. Cytokine. interleukin-17A

37. Hammerich L, Heymann F, Tacke F. Role of IL-17 and Th17 cells in liver diseases. Clin Dev Immunol. 2011;2011:345803.

38. Tang Y, Bian Z, Zhao L, et al. Interleukin-17 exacerbates hepatic steatosis and inflammation in non-alcoholic fatty liver disease. Clin Exp Immunol. 2011;166:281-90. 